\title{
A Neuro-Fuzzy based Control System for Solar Powered Wheelchair based on Feature Extraction of Surface EMG signal
}

\author{
M. Shamim Kaiser · Zamshed Iqbal Chowdhury · Shamim Al Mamun · Amir \\ Hussain · Mufti Mahmud
}

Received: 30-10-2015/ Accepted: date

\begin{abstract}
This paper presents the design and implementation of a low cost solar powered wheelchair for physically challenged people. The signals necessary to maneuver the wheelchair are acquired from different muscles of the hand using surface Electromyography (sEMG) technique. The raw sEMG signals are collected from the upper limb muscles which are then processed, characterized, and classified to extract necessary features for the generation of control signals to be used for the automated movement of the wheelchair. An artificial neural network based classifier is constructed to classify the patterns and features extracted from the raw sEMG signals. The classification accuracy of the extracted parameters from the sEMG signals is found to be relatively high in comparison to the existing methods. The extracted parameters used to generate control signals that are then fed into a microcomputer based control system (MiCS). A solar
\end{abstract}

M. Shamim Kaiser

Institute of Information Technology, Jahangirnagar University, Savar, 1342 - Dhaka, Bangladesh

Zamshed Iqbal Chowdhury

Institute of Information Technology, Jahangirnagar University, Savar, 1342 - Dhaka, Bangladesh

Shamim Al Mamun

Institute of Information Technology, Jahangirnagar University, Savar, 1342 - Dhaka, Bangladesh

Amir Hussain

University of Stirling, FK9 4LA - Stirling, Scotland, UK

Anhui University, Hefei, Anhui, 230601 - China

Mufti Mahmud ( $ه$ )

NeuroChip Lab, Department of Biomedical Sciences, University of Padova, 35131 - Padova, Italy

Institute of Information Technology, Jahangirnagar University, Savar, 1342 - Dhaka, Bangladesh

Theoretical Neurobiology \& Neuroengineering, University of Antwerp, 2610 - Wilrijk, Belgium

COSIPRA Lab, University of Stirling, FK9 4LA - Stirling, UK

E-mail: muftimahmud@ $\{$ juniv.edu, gmail.com $\}$ powered wheelchair prototype is developed and the above MiCS is introduced to control its maneuver using the sEMG signals. The prototype is then thoroughly tested with sEMG signals from patients of different age-groups. Also, the life cycle cost analysis of the proposed wheelchair revealed that it is financially feasible and cost effective.

\section{Introduction}

With the advent of sophisticated and stand alone computing platforms, human-machine control (HMC) interfaces have found applications in diverse fields including design of technology for physically disabled people $[1,2,3,4,5,6]$. The research on effective design of wheelchairs has been in the spotlight for over a decade now. During this period, the focus of the research has shifted from structural efficiency to ease of operation of the wheelchairs. The manual operations of wheelchairs require some forms of additional assistance-sometimes even from a care-giver which is not an option for everyone. The control systems for manually operated electric wheelchairs usually require a certain degree of external control inputs, such as in form of joystick or array of push buttons in order to provide required information (e.g., direction, speed, etc.) to the controller. Persons with marginal limbic ability are not expected to operate this kind of wheelchair by themselves. To overcome this problem, automated control based wheelchairs have been proposed and many research groups are still working on developing sophisticated and intelligent wheelchair control systems [7]. This kind of wheelchairs sample specific biological signals which are generated without extreme limbic movements, such as, Electromyogram (EMG) [8,9,10,11], Electroencephalogram (EEG) [4, 5, 6, 12], Electrooculogram (EOG) [13, 14], etc., to provide the necessary control signals for maneuvering the wheelchair. Further existing wheelchair 
control techniques include: eye gaze tracker method [15], eye blinking [16], tongue movement [17], speech recognition [18], and stereo omnidirectional system based gesture detection from images [19].

Due to the fact that these types of assistive devices (automatic wheelchairs) require very brief and impulsive signals (mentioned above) for their control systems to operate, they have been adopted by a wide range of physically challenged users. Since it is intended to use lesser degree of physical movements in controlling these assistive devices, the main challenge in designing such devices lies in the accurate and efficient extraction of all necessary information from the biological signals required to control those devices.

To this aim, intelligent systems have found to play a major role in design of wheelchair control systems. Specially, fuzzy logic controllers have been used in both microcontroller and field programmable gate array (FPGA) with ultrasonic sensors for controlling the wheelchair by taking dynamic timing information from the navigation environment [20]. Also, an FPGA based parallel fuzzy controller for wheelchair is proposed in [21]. The fuzzy system drives two H-bridges depending on the inputs provided by the disabled person.

In this work, a solar powered wheelchair is proposed which has it's control signals derived from a very common biological signal: the surface electromyogram (sEMG) signal. People with limited limbic abilities are the target users of this technology who have some abilities to produce certain level of muscular activation to generate the EMG signals and thereby to drive the wheelchair. An early prototype of such a wheelchair has been reported in [7].

\section{Methods}

Figure 1 shows the proposed solar powered wheelchair. The main components are: solar panels, battery charge controller, battery bank, sEMG signal acquisition and processing module (containing signal preprocessing, feature extraction, pattern classification), and wheelchair's motor control unit.

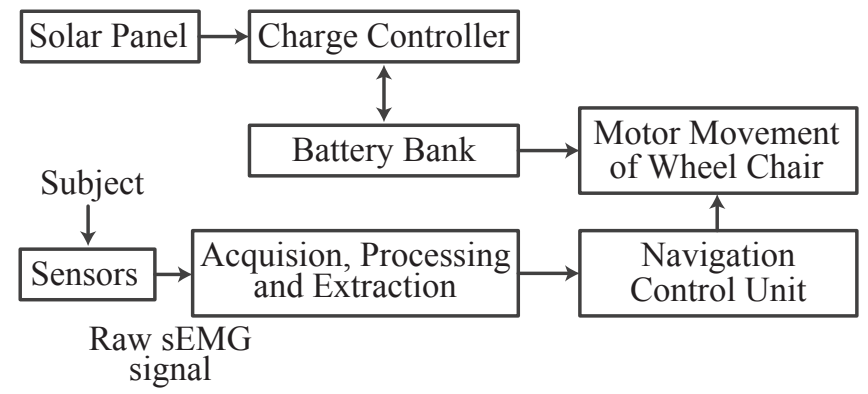

Fig. 1: Block diagram of the proposed wheelchair with it's various modules and communication flow among them.

\subsection{Wheelchair with Solar Power}

The custom-made wheelchair is seen in Fig. 2 with the design principles explained in the subsequent sections.

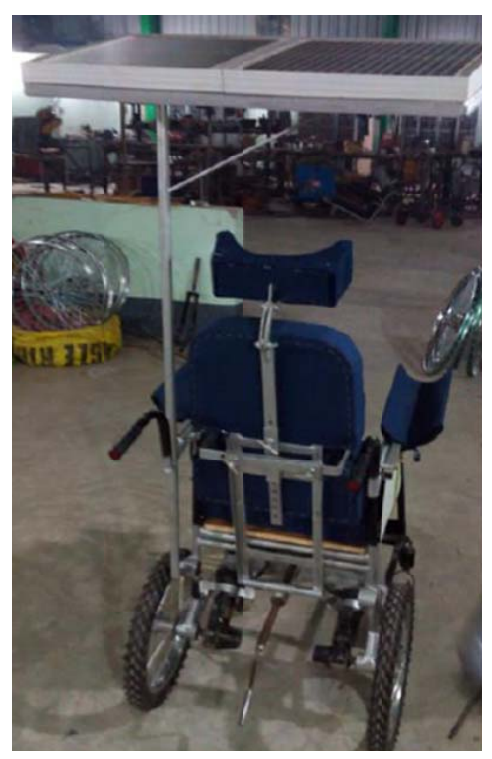

Fig. 2: Prototype of the proposed solar powered wheelchair.

Assembling of the Wheelchair The proposed wheelchair is a low cost one meaning that the designing and manufacturing of the wheelchair was carried out with locally available materials. Aluminum metal pipes which are considerably cheaper and lighter than steel were used. This type of material allowed the wheelchair to be light in weight, thereby reducing the required driving force. Also, it was designed as tilted-seat wheelchair facilitating the usability for patients with spinal cord injury.

The battery is housed just under the seat of wheelchair, utilized photovoltaic (PV) energy for charging which saved considerable amount of utility bill in comparison to the ones charged using the national grid.

Additionally, the control system was designed by means of a powerful Advanced RISC Machine (ARM) processor which allowed complex algorithms to be executed, thus, avoiding expensive hardware to be employed in the design.

In comparison to other existing automatic wheelchair systems, the attribute 'low cost' is justified considering the technological advantages in parallel with the price of the product. The average price of an automatic battery powered wheelchair is around US\$2500. A vast majority of them are designed with a focus on structural privileges such as folding ability, light weight battery etc. as well as energy concerns such as low power consumption. The technological features of these wheelchairs are limited to providing external input via joystick or keypad. Considering those limitations, the 
flexibility provided by the proposed automatic wheelchair is greater with a manufacturing cost of around US\$950.

Interfacing with the Solar Panel Solar panels (IM60, Motech Industries Inc., Taiwan) were used to convert solar radiation into electrical energy. Energy from sunlight was stored in battery bank, which was controlled by a commercially available charge controller (20A 12V/24V Auto Switch MPPT Solar Panel Battery Regulator Charge Controller KJ) to track the maximum power point (MPP). Lead acid battery (36B20L, Hamco Corporation, Dhaka, Bangladesh) was considered because of its low cost, reliability, tolerance to overcharge, low initial impedance, and the ability to deliver high current. The battery bank was utilized as backup energy provider because the PV system only generates enough electricity with sunlight.

\subsection{Surface Electromyogram (sEMG) Signals}

EMG signal is generated due to the rapid movement of ions across a cell membrane causing a charge imbalance between inside and outside of the membrane. Due to the diffusion of $\mathrm{Na}^{+}, \mathrm{K}^{+}$and $\mathrm{Cl}^{-}$ions, a potential difference is generated and propagates through the muscle fiber in form of an Action Potential (AP). This potential ranges from a resting value of $-90 \mathrm{mV}$ to a peak value of $+35 \mathrm{mV}$ and the instantaneous potential is directly proportional to the force produced by contraction of muscle fibers conducting the AP [22,23]. The sEMG is measured as the potential difference between a pair of electrodes with an additional electrode as reference. Since the measured potentials are very low in amplitude, the signals require amplification and preprocessing prior to be used in the control circuitry.

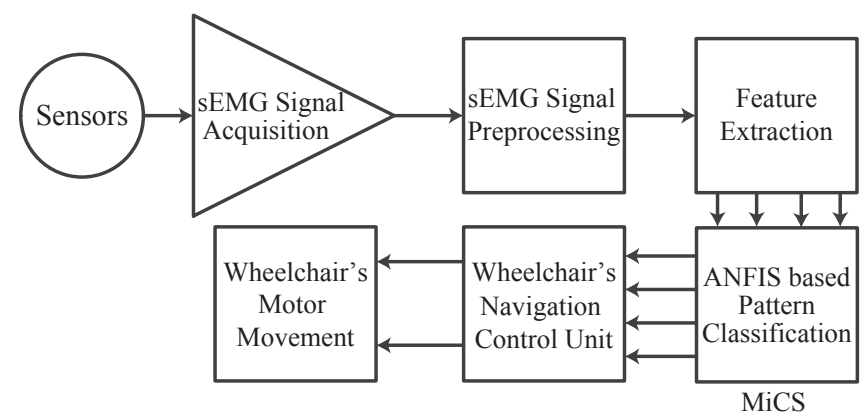

Fig. 3: Block diagram of the wheelchair's control system with sEMG signal.

The acquired sEMG signals (see sec. 2.3 for acquisition process) were preprocessed and some important features were extracted (see sec. 2.4 for feature extraction process) from its time and frequency domains. The time do- main features were: mean absolute value (MAV) and slope sign change (SSC) [24,25]. Whereas, the frequency domain features were: zero crossing (ZC) and local minima (LM) [26]. These features were further classified using an Adaptive Neuro-Fuzzy Interface System (ANFIS) (see sec. 2.5 model for creating the control signals which were then fed into the driving circuit for the control of the wheelchair's motors. The control diagram of the wheelchair is shown in Fig 3 .

\subsection{Signal Acquisition and Processing}

Hand muscle movements of a subject generate sEMG signals. The signals were taken during four different hand motions: wrist extension (WE), wrist flexion (WF), thumb movement $(\mathrm{T})$ and finger movement except thumb (F). Appropriate positions for the lead electrodes were found using the trial and error method. The signals were collected for 1 minute at $1 \mathrm{kHz}$ sampling frequency.

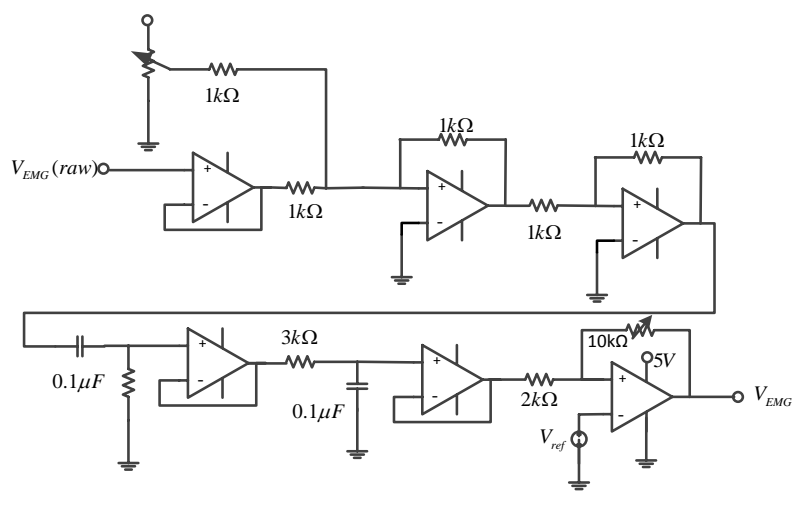

Fig. 4: Circuit diagram of sEMG signal acquisition.

The signal acquisition system front-end used in acquiring the sEMG signals is shown in Fig. 4 The acquired signals are preprocessed, filtered and amplified. Surface EMG sensor of identical electrical characteristics were used to acquire muscular signals, denoted by $V_{E M G}$. The frequency range of the sEMG signals lie between 7 and $500 \mathrm{~Hz}$ [27. 28].

Due to the fact that the sEMG signals are very often contaminated by noise from human body motion, electrode contact problems, power line, amplifier etc., they might not produce quality signals to be used to control the wheelchair. To mitigate the noise effects, a number of design issues were considered during the design phase of this work. Starting with placing the stimulation electrodes in proper places on the body with respect to recording electrodes. After acquisition of the raw sEMG signals, they are passed through a 
system which pre-processes those raw sEMG signals to improve the signal-to-noise ratio (SNR).

The motion artifacts were eliminated by the bandpass filtering the signals between $10 \mathrm{~Hz}$ and $20 \mathrm{kHz}$. The electrode noise, power line noise etc., were difficult to remove, however, the sEMG signals were denoised (rather the SNR was improved) by wavelet-based methods as described in [29,30]. These denoised and high SNR sEMG signals were further processed to extract important features.

\subsection{Feature Extraction}

In general, feature extraction from the sEMG signals is complex and very important for classification of the hand movements. Many researchers use time-frequency domain technique for feature extraction. Below is a list containing the heuristically selected features.

- Time domain (as suggested in [24, 25]):

- Mean absolute value (MAV): the MAV was calculated using the formula

$M A V=\frac{1}{N} \sum_{n=1}^{N}\left|x_{n}\right|^{2}$

- Slope sign change (SSC): the SSC was calculated using the formula

$$
\begin{aligned}
& S S C=\sum_{n=2}^{N-1}\left[f\left[\left(x_{n}-x_{n+1}\right) \times\left(x_{n}-x_{n-1}\right)\right]\right] \\
& f(x)=1 \text { if } x \geq \text { threshold, else } 0
\end{aligned}
$$

- Frequency domain (as suggested in [26]):

- Zero crossing (ZC): the ZC was calculated using the equation

$Z C=\sum_{N-1}^{n=1}\left[\operatorname{sgn}\left(x_{n} \times x_{n+1}\right) \cap\left|x_{n}-x_{n-1}\right| \geq\right.$ threshold $]$ (3)

- Local minima (LM): if $x_{i-1}>x_{i}$ and $x_{i}<x_{i+1}$ where $1<i<n-1$, index $i$ is a local minima index and its local minimum value is $x_{i}$. If $\left(x_{n-1}<x_{n+1}\right)$ it is right minimum, else if $\left(x_{n-1}>x_{n+1}\right)$ it is left minimum.

The calculation of $M A V, S S C$, and $Z C$ was performed as described in [31], and the LM was calculated as in [32]

\subsection{Microcomputer based Control System (MiCS)}

Fuzzy Inference System (FIS) was first introduced in 1965 [33]. Fuzzy logic can incorporate human decision making capability in the form of IF-THEN rules. The sEMG signals are non-stationary in nature, thus the pattern classifications are very difficult. Fuzzy logic system is able to classify the pattern of biological signals. In FIS, inputs are fuzzified to a value that lies in the interval $[0,1]$. Then it is interpreted by the IF-THEN rules [33].
The Adaptive Neuro-Fuzzy Inference System (ANFIS) for this control system was implemented in a Raspberry Pi 2 (https://www.raspberrypi.org/) and the necessary codings were done in python (v.3.4, https: / / www. python.org/).

The singular value decomposition method was used to determine the number of fuzzy rules. The membership parameters of the Sugeno type ANFIS were adjusted by using neural network [34]. Fig. 5] shows the ANFIS structure with five inputs, denoted by $I_{i p}^{J}$, where $p=[M A V, S S C, A R, Z C$ and $L M]$ and one output, denoted by $O_{i}[34]$. Bell shaped membership functions $(M F)$ were used [34] and the required parameters for the input-output pairs were calculated using hybrid back propagation and LMS algorithms. Using membership functions, four fuzzy rules were derived. The output of the system can be written as in Eq. 4

$O=\sum_{i=1}^{L}\left[\frac{\prod_{j=1}^{n} M F_{i}^{j}\left(I_{i}^{j}\right)\left(Z_{i}\right)}{\sum_{i=1}^{L}\left(\prod_{j=1}^{n} M F_{i}^{j}\left(I_{i}^{j}\right)\right)}\right]$ 5

The output of each rule, denoted by $Z_{i}$, was given by Eq.

$Z_{i}=\alpha_{i_{1}} I_{1}+\alpha_{I_{2}} I_{2}+\alpha_{I_{3}} I_{3}+\alpha_{I_{4}} I_{4}+\alpha_{I_{5}}+I_{5}+\alpha_{I_{6}}$

where $\alpha_{i j}$ is the consequent parameters of input $j$. The linguistic variable of the inputs are $M F_{i}^{1}=$ low $M F_{i}^{2}=$ average and $M F_{i}^{3}=$ high.

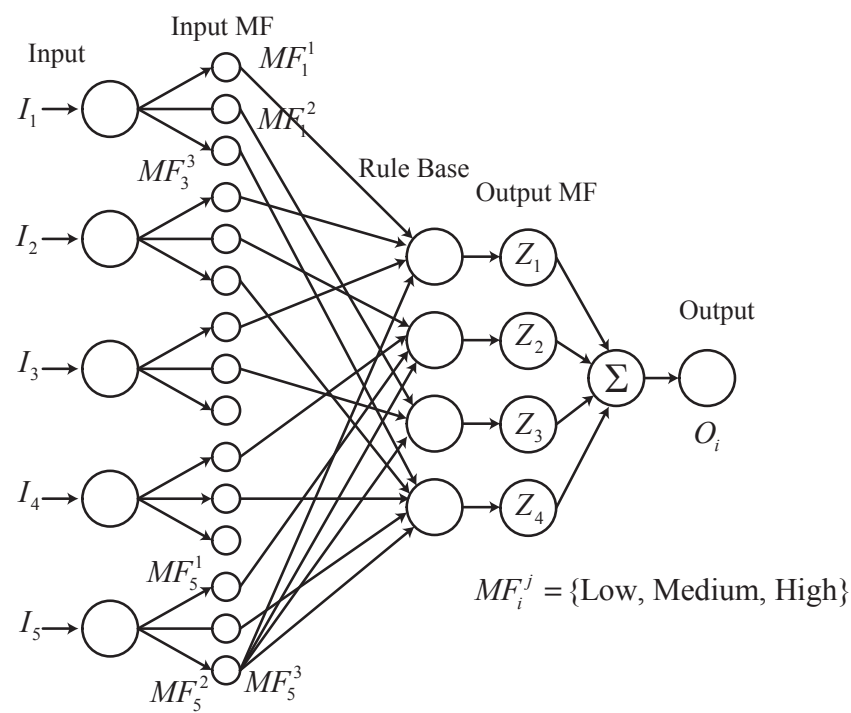

Fig. 5: Structure of adaptive neuro-fuzzy inference system with five input and 1 output. $I$ : Input, $M F$ : Membership Function, $O$ : Output.

The multiplexed output MF's of the ANFIS were then fed to the direction control circuit for controlling the wheelchair's motors. 


\subsection{Wheel Chair's Direction Controller}

Fig. 6illustrates control circuit for driving the wheelchair in a particular direction. Here $D$ refers to diode (1N5400) and $T$ refers to transistor (2SD1760). The diodes $D_{1}$ and $D_{2}$ were used to discharge the residual current when motors were in OFF state.

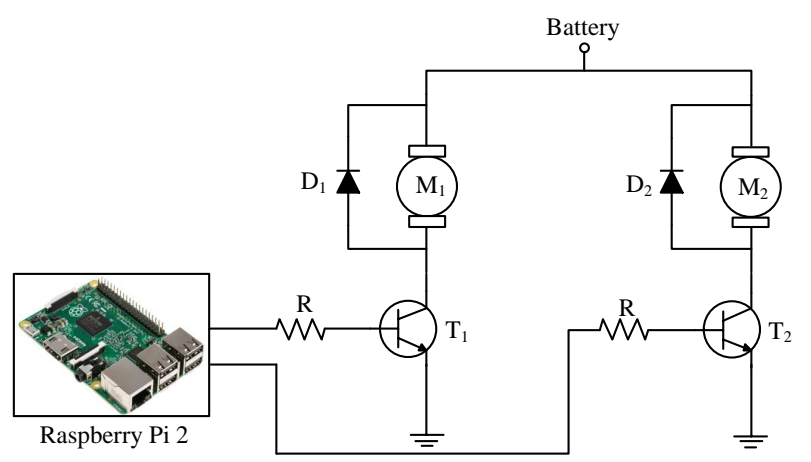

Fig. 6: Wheel chair direction control circuit

The direction control strategy for the proposed wheelchair is given in algorithm 1. Initially, the wheel chair was stalled in its position and waited for a control signal (CS). One of the four CSs were received from the MiCS: Forward CS (FCS); Left CS (LCS); Right CS (RCS); Stop CS (SCS).

When the received control signal was FCS, both $T_{1}$ and $T_{2}$ were switched ON. Thus motors (M1 and M2) moved the wheelchair in the forward direction. If the received control signal was LCS, $T_{1}$ and $T_{2}$ were switched OFF and ON, respectively, to move the wheelchair in leftward direction. Similarly, if the received control signal was RCS, $T_{1}$ was switched ON while $T_{2}$ was OFF, this caused the wheelchair to move in rightward direction. When the SCS was received, both $T_{1}$ and $T_{2}$ were switched OFF and the movement of the wheelchair was halted.

\subsection{Financial Feasibility Matric: LCC}

To evaluate the financial feasibility of the proposed wheelchair, the life cycle cost (LCC) analysis of the entire system was performed using Eq. 6 The LCC was computed on the capital cost, total operation and maintenance cost, and total replacement cost over the whole life time. Based on [7], LCC was used to find the optimal energy configuration of the system.

$L C C=C_{c}+\sum_{n=1}^{N} C_{o \& m}^{n}+\sum_{n=1}^{N} C_{r}^{n}$

where $N$ is the life time of the system, $C_{c}$ is the capital cost of the system, $C_{o \& m}^{n}$ is the annual operation and maintenance cost of year $n$ and $C_{r}^{n}$ is the replacement cost of the system.

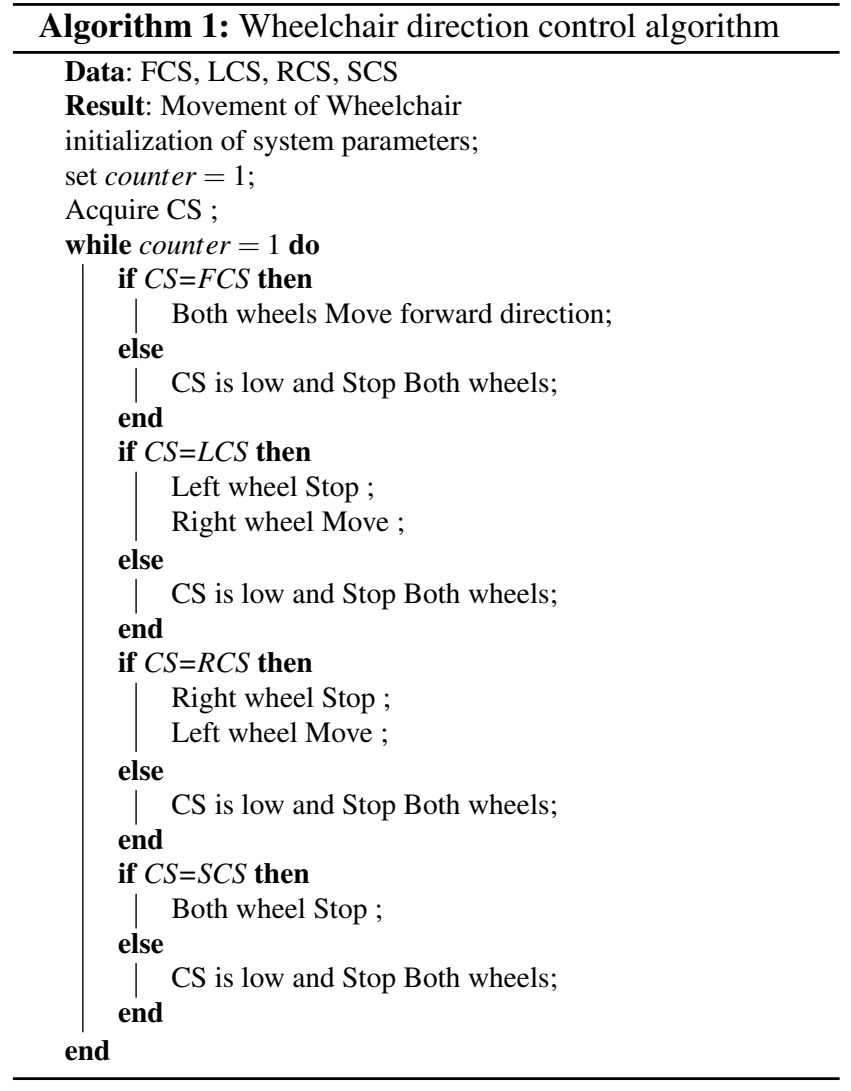

\section{Results and Discussion}

3.1 Feasibility of Wheelchair Maneuvering using sEMG Signals from Hand Movements

The wheelchair was tested with sEMG signals recorded from the hand muscles using sEMG sensors. Table 1 shows hand movements, sensor position on different muscles to extract sEMG signals and the corresponding functions for the wheelchair. The mean value of achieved accuracy was found to be high in comparison to the results presented in our previous work [7].

Table 1: Function classification accuracy by ANFIS.

\begin{tabular}{|c|c|c|c|c|}
\hline \multirow[b]{2}{*}{ Motion } & \multirow[b]{2}{*}{ Active Muscles } & \multirow[b]{2}{*}{ Function } & \multicolumn{2}{|c|}{ Accuracy } \\
\hline & & & Kaiser et al. & Proposed \\
\hline WE & $M_{1}$ & Break & $91.0 \%$ & $97.5 \%$ \\
\hline WF & $M_{2}$ & $\begin{array}{l}\text { Move } \\
\text { forward }\end{array}$ & $94.0 \%$ & $97.5 \%$ \\
\hline $\mathrm{T}$ & $M_{3}$ & Left/Right & $93.1 \%$ & $94.0 \%$ \\
\hline $\mathrm{T}$ & $M_{4}$ & Left/Right & $93.1 \%$ & $94.0 \%$ \\
\hline $\mathrm{F}$ & $M_{5}$ & Left/Right & $93.2 \%$ & $94.0 \%$ \\
\hline
\end{tabular}


The sEMG signals were successfully recognized for their related movements. Table 2 shows the confusion matrix of the identified and real movements of the hand obtained from MiCS. It can be appreciated that during the classification process, the MiCS could successfully distinguish between the movements from the wrist and the fingers. Also, the accuracy of identifying the wrist movements $(97.5 \%)$ were higher than that of the finger movements $(96 \%)$. The maximum recognition rate was found to be $97.5 \%$ with maximum error to be $4 \%$.

Table 2: Recognition of hand movements by MiCS.

\begin{tabular}{lllll}
\hline Identified Real & WE & WF & T & F \\
\hline WE & $97.5 \%$ & $2.5 \%$ & $0 \%$ & $0 \%$ \\
WF & $2.5 \%$ & $97.5 \%$ & $0 \%$ & $0 \%$ \\
T & $0 \%$ & $0 \%$ & $96 \%$ & $4 \%$ \\
F & $0 \%$ & $0 \%$ & $4 \%$ & $96 \%$ \\
\hline
\end{tabular}

Table 3 shows accuracy comparison of hand movements' recognition among three studies:

1. From Nishikawa etal. [35],

2. From Kehzri \& Jahed [26], and

3 . From the system proposed in this work.

The achieved accuracy of the proposed approach is found to be better than that of the previous studies. Its worth mentioning here that this approach requires less amount of hand movements to achieve such high accuracy in comparison to other studies.

Table 3: Accuracy comparison of hand movements' recognition in different studies.

\begin{tabular}{lll}
\hline Selected Study & $\begin{array}{l}\text { Hand move- } \\
\text { ments }\end{array}$ & Average accuracy (\%) \\
\hline Nishikawa etal. $[35]$ & 10 & 92.10 \\
Kehzri \& Jahed [26] & 6 & 96.70 \\
Proposed system & 4 & 96.85 \\
\hline
\end{tabular}

\subsection{Financial Feasibility}

LCC is a useful tool for understanding the cost-effectiveness of different types of wheelchairs. For a wheelchair, LCC calculation is performed considering the expected lifetime of the system to be 20 years. Since the battery lifetime is five years, during the lifetime of the system the battery should be replaced at least three times (Non-recurring cost). Table 4 shows LCC analysis for different wheelchairs available in the global market. As evidentiated, the solar powered wheelchair proposed in this work is financially feasible.
Table 4: LCC analysis (in USD).

\begin{tabular}{|c|c|c|c|}
\hline Type & Components & Cost & $\begin{array}{l}\text { Total } \\
\text { LCC }\end{array}$ \\
\hline \multirow{9}{*}{$\begin{array}{l}\text { Solar Powered } \\
\text { Wheelchair }\end{array}$} & PV Array & $\$ 100$ & \\
\hline & Wheelchair & $\$ 77$ & \\
\hline & Battery & $\$ 285$ & \\
\hline & Converter & $\$ 64$ & \$ 997 \\
\hline & Other Components & $\$ 50$ & \\
\hline & Installation Cost & $\$ 26$ & \\
\hline & Electricity Cost & $\$ 40$ & \\
\hline & $\mathrm{O}$ and $\mathrm{M}$ Cost & $\$ 38$ & \\
\hline & Non-recurring cost & $\$ 320$ & \\
\hline \multirow{4}{*}{$\begin{array}{l}\text { Compass } \\
\text { GP605 }\end{array}$} & Wheelchair & $\$ 3,399$ & $\$ 3907$ \\
\hline & Electricity Cost & $\$ 150$ & \\
\hline & $\mathrm{O}$ and $\mathrm{M}$ Cost & $\$ 38$ & \\
\hline & Non-recurring cost & $\$ 320$ & \\
\hline \multirow[t]{4}{*}{ KD Smart Chair } & Wheelchair & $\$ 1,995$ & $\$ 2503$ \\
\hline & Electricity Cost & $\$ 150$ & \\
\hline & $\mathrm{O}$ and $\mathrm{M}$ Cost & $\$ 38$ & \\
\hline & Non-recurring cost & $\$ 320$ & \\
\hline \multirow{4}{*}{$\begin{array}{l}\text { LiteRider } \\
\text { Portable }\end{array}$} & Wheelchair & $\$ 1,999$ & $\$ 2507$ \\
\hline & Electricity Cost & $\$ 150$ & \\
\hline & $\mathrm{O}$ and $\mathrm{M}$ Cost & $\$ 38$ & \\
\hline & Non-recurring cost & $\$ 320$ & \\
\hline
\end{tabular}

\section{Conclusion}

A solar powered electric wheelchair was proposed and a prototype was developed in order to test the desired functionalities. The control sequences required to maneuver the wheelchair was derived from sEMG signals. The raw signals were carefully preprocessed to remove the artifacts present in the signals in order to improve the SNR. The improved signals were fed to MiCS, a powerful stand alone microcomputer based control system, which took the muscle activation information and applied a feature extraction algorithm to extract useful features representing the hand movements. Later, an adaptive neuro-fuzzy interface system was applied to classify and identify the hand movements and translate them to desired direction parameters. The MiCS yielded a satisfactory accuracy of above $97 \%$ in deciphering the hand movements from the sEMG signals. The direction parameters generated by MiCS were then used in driving the wheelchair. The design of the system was flexible enough, thanks to the functionalities performed by MiCS, for the integration of additional features such as global positioning system based location, usage of EEG instead of EMG, speech based emergency brake, etc., which are left to be implemented in the future. 
Conflict of Interest Statement: The authors declare that the research was conducted in the absence of any commercial or financial relationships that could be construed as a potential conflict of interest.

Authors and Contributors: This work was carried out in close collaboration between all co-authors. MSK, ZIC, SAM, and MM first defined the research theme and contributed an early design of the system. MSK, ZIC, and SAM further implemented and refined the system development. MSK, ZIC, SAM, MM, and AH wrote the paper. All authors have contributed to, seen and approved the final manuscript.

Ethical Approval: All procedures performed in studies involving human participants were in accordance with the ethical standards of the institutional and/or national research committee and with the 1964 Helsinki declaration and its later amendments or comparable ethical standards.

Informed Consent: Informed consent was obtained from all individual participants included in the study.

\section{References}

1. Lebedev MA, Nicolelis MA. Brain-machine interfaces: past, present and future. Trends Neurosci. 2006;29(9):537-46.

2. Hochberg LR, Serruya MD, Friehs GM, Mukand JA, Saleh M, Caplan AH, Branner A, Chen D, Penn RD, Donoghue JP. Neuronal ensemble control of prosthetic devices by a human with tetraplegia. Nature. 2006;442(7099):164?71.

3. Kubler A, Kotchoubey B, Kaiser J, Wolpaw J, Birbaumer N. Brain-computer communication: unlocking the locked in, Psychol Bull, 2001; 127(3):358-375.

4. Mahmud M, Bertoldo A, Vassanelli S. EEG Based BrainMachine Interfacing: Navigation of Mobile Robotic Device. in Mobile Robots - Control Architectures, Bio-Interfacing, Navigation, Multi Robot Motion Planning and Operator Training, J. B dkowski, Ed. Rijeka, Croatia: InTech, 2011:129 - 144. ISBN: 978953-307-842-7.

5. Mahmud M, Hawellek D, Bertoldo A. EEG based brain-machine interface for navigation of robotic device. in Proc. 3rd IEEE/RASEMBS International Conference on Biomedical Robotics and Biomechatronics (BioRob2010), Tokyo, Japan, 2010:168-172.

6. Mahmud M, Hawellek D, Valjamae A. A brain-machine interface based on EEG: extracted alpha waved applied to mobile robot. in Proc 2009 ECSIS Symposium on Advanced Technologies for Enhanced Quality of Life (AT-EQUAL 2009), Iasi, Romania, 2009:28-31.

7. Kaiser MS, Chowdhury ZI, Al Mamun S, Hussain A, Mahmud M. Solar Powered Wheel Chair For Physically Challenged People Using Surface EMG Signal. In: Proceedings of the 2015 IEEE Symposium on Computational Intelligence in healthcare and ehealth (IEEE CICARE 2015). Cape Town, South Africa, 2015.

8. Rani P, Sarkar M. EMG-based high level human-robot interaction system for people with disability. Proceedings of 2005 IEEE International Workshop on Robot and Human Interactive Communication (ROMAN 2005), Nashville, Tennessee. 2005:280-285.

9. Artemiadis P, Kyriakopoulos K. EMG-based teleoperation of a robot arm in planar catching movements using ARMAX model and trajectory monitoring techniques. Proceedings 2006 IEEE International Conference on Robotics and Automation (ICRA 2006), Orlando, Florida. 2006:3244-3249.

10. Artemiadis P, Kyriakopoulos K. Teleoperation of a robot manipulator using EMG signals and a position tracker. Proceedings of
2005 IEEE/RSJ International Conference on Intelligent Robots and Systems (IROS 2005), Alberta, Canada. 2005:1003-1008.

11. Moon I, Lee M, Chu J, Mun M. Wearable EMG-based HCI for Electric-Powered Wheelchair Users with Motor Disabilities. in Robotics and Automation, 2005. ICRA 2005. Proceedings of the 2005 IEEE International Conference on. 2005, 2649-2654, doi: 10.1109/ROBOT.2005.1570513.

12. Tanaka K, Matsunaga K, Wang HO. ElectroencephalogramBased Control of an Electric Wheelchair. in Robotics, IEEE Transactions on , 2005;21(4):762-766, doi: 10.1109/TRO.2004.842350.

13. Barea R, Boquete L, Mazo M, Lopez E, Bergasa LM. EOG guidance of a wheelchair using neural networks. in Pattern Recognition, 2000. Proceedings. 15th International Conference on , 2000;4:4668-4671, doi: 10.1109/ICPR.2000.903006.

14. Arai K, Mardiyanto R. Eye-Gesture Controlled Intelligent Wheelchair using Electro-Oculography. International Journal of Advanced Computer Science and Applications, 2011;2(12).

15. Nguyen QX, Jo S. Electric wheelchair control using head pose free eye-gaze tracker. Electron Lett 2012; doi: 10.1049/el.2012.1530.

16. Purwanto D, Mardiyant R, Arai K. Electric wheelchair control with gaze direction and eye blinking. Artif Life Robotics 2009. doi: 10.1007/s10015-009-0694-X.

17. Huo X, Wang J, Ghovanloo M. Wireless control of powered wheelchairs with tongue motion using tongue drive assistive technology. In: Conf Proc IEEE Eng Med Biol Soc. 2008;2008:4199202. doi: 10.1109/IEMBS.2008.4650135.

18. Fezari M, Bousbia-Salah M, Bedda M. Speech and sensor in guiding an electric wheelchair. in Computational Intelligence Methods and Applications, 2005 ICSC Congress on, 2009, doi: 10.1109/CIMA.2005.1662320.

19. Satoh Y, Sakaue K. An Omnidirectional Stereo Vision-Based Smart Wheelchair. EURASIP Journal on Image and Video Processing 2007, doi:10.1155/2007/87646.

20. Ponce PC., Rodrguez R J, Segura AN. A Novel Fuzzy Controller Switching Frequency of an Induction Motor Using Direct Torque Control, PES 2002, Crete, Greece.

21. Poplawski M, Bialko M. "Implementation of parallel fuzzy logic controller in FPGA circuit for guiding electric wheelchair," in $\mathrm{Hu}$ man System Interactions, 2008 Conference on , 2008:405-408, doi: 10.1109/HSI.2008.4581473.

22. Mouri J. EEG-based Analysis for the Design of Adaptive rain Interfaces. $\mathrm{PhD}$ thesis, Universitat Politcnica de Catalunya, Barcelona, Spain; 2003.

23. Santis AD, Iacoviello D. Robust real time eye tracking for computer interface for disabled people. Comput. Methods Progr. Biol. 2009;96:1-11.

24. Englehart K, Hudgins B, Parker PA, Stevenson M. Classification of the myoelectric signal using time-frequency based representations. Medical Engineering and Physics. Elsevier Science. 1999;21:431-438.

25. Karlik B. Differentiating Type of Muscle Movement via AR Modeling and Neural Network Classification. Turk J Elec Engin. 1999;7(1):45-52.

26. Khezri M, Jahed M. Real-time intelligent pattern recognition algorithm for surface EMG signals. Biomed Eng Online. 2007; 6:112 doi: 10.1186/1475-925X-6-45.

27. Kash SA, Menon A, Gupta A, Wakeel MW, Praveen MN, Meena P. A novel strategy for controlling the movement of a smart wheelchair using internet of things. in Global Humanitarian Technology Conference - South Asia Satellite (GHTC-SAS), 2014 IEEE , 2014:154-158, doi: 10.1109/GHTC-SAS.2014.6967575

28. Krishnan JR Hemalatha RJ; Umashankar G, Ahmed N, Nayak SR. Development of Magnetic Control System for Electric Wheel Chair Using Tongue. Series Advances in Intelligent Systems and Computing, 2014;308:635-641 
29. Han JS, Bien Z, Kim DJ, Lee HE, Kim JS. Human-machine interface for wheelchair control with EMG and its evaluation. Engineering in Medicine and Biology Society,Proceedings of the 25th Annual International Conference of the IEEE 2003;2:1602-1605, doi: 10.1109/IEMBS.2003.1279672.

30. Tsui CSL, Jia P, Gan JQ. Huosheng Hu; Kui Yuan, EMG-based hands-free wheelchair control with EOG attention shift detection. Robotics and Biomimetics, IEEE International Conference on 2007. 2007:1266-1271, doi: 10.1109/ROBIO.2007.4522346.

31. Shroffe DEH, Manimegalai P. Hand gesture recognition based on EMG signals using ANN. international journal of computer application. 2013;3(2):31-39.

32. Hu Z, Cai Z. Feature Synthesis Algorithm Combined with k-NN Classifier for Spectral Data Classification. Communications in Computer and Information Science. 2010;107:254-263.

33. Zadeh LA. Fuzzy Sets. Information and Control. 1965,8:338-352.

34. Kaiser MS, Chaudary H, Shah MRA, Ahmed KM. Neuro-Fuzzy (NF) based relay selection and resource allocation for cooperative networks. International Conference on Electrical Engineering/Electronics Computer Telecommunications and Information Technology (ECTI-CON), 2010: 244-248.

35. Nishikawa D, Yu W, Yokoi H, Kakazu Y. On-line learning method for EMG prosthetic hand control. Electron. Comm. Jpn. Pt. III, 2001;84:35-46. doi: 10.1002/ecjc. 1040

This is a postprint of an article published in Cogn. Comput. The final version is available at: https://doi.org/10.1007/s12559-016-9398-4 Cite as: M.S. Kaiser, Z.I. Chowdhury, S. Al Mamun, A. Hussain, M. Mahmud. (2016). A Neuro-Fuzzy Control System Based on Feature Extraction of Surface Electromyogram Signal for Solar-Powered Wheelchair. Cogn. Comput., 8(5): 946-954. doi: 10.1007/s12559-016-9398-4. 